Conclusion We still have lots to learn but the Strategy Balanced Scorecard System has proven a crucial tool in transforming our hospice's approach to strategy and planning and in building a 'One Hospice' culture based on and driven by our shared values.

\section{P-161 HOPE FOR THE BEST, PLAN FOR THE WORST? IMPROVING RESILIENCE WITH BUSINESS CONTINUITY PLANNING}

Laura Brisley, Kevin McGill. St Helena, Colchester, Essex

10.1136/spcare-2021-Hospice. 177

Background The 2009 Flu pandemic underlined to health care providers the importance of an effective business continuity plan (Hopkin, 2018), and was further highlighted by Brexit and COVID-19. Organisations face increasing ranges of external risks and threats (Copeman, 2017), showing the need for competent risk management. Business continuity planning (BCP) is an integral part of the risk management process, and hospices need to be able to actively demonstrate their resilience.

Aim A review of current resilience was undertaken, with the aim of developing and implementing a fit for purpose Business Continuity Plan (NHS Commissioning Board, 2013), which could be tested against multiple scenarios, so the organisation's 'state of preparedness' for a range of service disruptive scenarios can be regularly challenged and improved.

Method A workshop was organised to facilitate discussion around our current resilience and identify gaps of existing arrangements. A new BCP policy was created along with a BCP implementation plan. Focussed team discussions were undertaken, where each service explored their key functions and highlighted necessary emergency supply items, which were procured and made available. Familiarisation training was incorporated into team meetings organisation-wide. External critical friends were invited to review the new documentation and provide feedback to further improve our BCP processes and procedures.

Results The organisation's new Business Continuity Plan is now operational and has been reviewed and updated as a result of COVID-19. Our journey has been shared with hospices to help improve overall hospice awareness of risk and resilience, and promote best practice within and without our own organisation.

Conclusion The BCP review created a more aware and dynamic organisational approach to risk management. Furthermore, as funding and workforce challenges continue to cast uncertainty for hospices, our organisation now recognises resilience is an ongoing, fluid process which requires continual review and challenge to remain effective, and a co-ordinated and multi-disciplinary team approach is required to assure an effective and practical BCP.

\section{P-162 PROJECT ECHO - ADDRESSING THE NEED FOR SUPPORTIVE AND HIGH QUALITY PALLIATIVE CARE EDUCATION}

${ }^{1}$ Janet Diffin, ${ }^{1}$ Tracey McTernaghan, ${ }^{2}$ Aine McMullan, ${ }^{2}$ Martin Hayes, ${ }^{1}$ Max Watson, ${ }^{1}$ Christopher Jenkins. ${ }^{1}$ Hospice UK, Belfast, UK; ${ }^{2}$ Health and Social Care Board, Belfast, UK

10.1136/spcare-2021-Hospice.178
Background Palliative care requires an integrated, multidisciplinary approach and involves a range of specialities. Despite increasing demand, limited resources are often available to deliver high quality palliative care education and support that is tailored to meet the needs of healthcare professionals. Project ECHO (a tele-mentoring programme) could overcome this gap by using video-conferencing to deliver best practice guidance and education from specialists, and case-based learning with peer discussion. Five ECHO networks in Northern Ireland were established (Cardiology/Heart Failure, District nurses, Community pharmacy, Paediatrics, Marie Curie registered nurses). Participants set the curriculum, network aims and objectives, which included increasing participants' palliative care knowledge and skills, enhancing confidence, building relationships, and facilitating peer support. 45 ECHO sessions were delivered across five networks between 2018-2019 with 194 participants attending $\geq 2$.

Aim The aim of evaluation was to identify if each network achieved its objectives.

Methods Retrospective online survey issued to participants after the final ECHO.

Results 27\% (60/224) registered participants responded. ECHO was identified as a suitable model for education delivery and valued for case-based learning. Each network met their objectives: increased knowledge and management of palliative care patients. ECHO was identified as a suitable model for delivering education and the opportunity for case-based learning was valued. Impacts on practice included improvements in, inter-agency working, networking, communication with patients and families, confidence to make clinical decisions. Professional isolation was also reduced. Barriers to participation included time restraints due to staff shortages or workload.

Conclusion Project ECHO may be ideal for delivering palliative care education and support across multiple settings. However, protected time for participation is recommended.

\section{P-163 PROJECT ECHO BRINGING OUR HOSPICES CLOSER TOGETHER AND SUPPORTING THE CARE COMMUNITY}

Chris Charters. St Leonard's Hospice, York, UK

\subsection{6/spcare-2021-Hospice. 179}

Project ECHO is a case based, collaborative learning tool designed to enable health and social care professionals to connect with each other. Originating in the USA and taking place primarily on the Zoom platform; it aims to allow staff to share their knowledge and skills with each other to form a community of practice. The key ethos of everyone being both a learner and a teacher enables staff to benefit from a collective learning experience and reduce any isolation that they may feel in their role.

Since January 2020, St Catherine's and St Leonard's Hospices have formed a collaborative Project ECHO hub, through NHS England funding secured by the Humber Coast and Vale STP. The hospices and key stakeholders work closely to deliver education to the regional health and social care communities.

Outputs address identified need across the region. Work with local CCGs in spring/summer 2020 supported the care provider community with regular forums and education, 